\title{
Controllable synthesis of gold nanoparticles in aqueous solution by microwave assisted flow chemistry
}

\author{
Mustafa K Bayazit, Jeffrey Yue, Enhong Cao, Asterios Gavriilidis and Junwang Tang* \\ Department of Chemical Engineering, University College London, Torrington Place, \\ London WC1E 7JE, United Kingdom
}

\begin{abstract}
The development of energy efficient, reproducible and high throughput approaches to gold nanoparticles (Au-NPs) synthesis have gained increasing attention over the past decades due to their application in biomedicine, sensor and catalysis. In this work, single mode microwave irradiation is for the first time combined with microflow chemistry to fabricate Au-NPs continuously and reproducibly with controllable size in an aqueous solution. The major experimental parameters including microwave power, citrate-to-gold molar ratio ([Cit]/[Au]) and reaction residence time have been investigated systematically. As indicated by TEM, the mean particle width of the synthesised Au-NPs is between 4 and $15 \mathrm{~nm}$ with mean aspect ratio between $\sim 1.4$ and 2.2 after only $90 \mathrm{~s}$ microwave irradiation. Furthermore the Au particle morphology can be manipulated from nanowires to nanoparticles by adjusting the $[\mathrm{Cit}] /[\mathrm{Au}]$ ratio. HRTEM analysis of the produced Au-NPs and UV-vis spectroscopy suggest a correlation between the red-shifted surface plasmon resonance peak and the particle aspect ratio that is probably dependent on the creation of particle-particle junctions, which alter both the size and the aspect ratio of the Au-NPs. The synthesis further demonstrated high reproducibility.
\end{abstract}

Keywords: microwave, flow chemistry, gold nanoparticles, particle size, aspect ratio 


\section{Introduction}

Gold nanoparticles (Au-NPs) have attracted increasing attention over the last decades due to their unique surface plasmon resonance properties, making them suitable in many areas such as healthcare and sensing applications. ${ }^{1,2}$ Reduction of $\mathrm{HAuCl}_{4}$ in boiling water by sodium citrate $\left(\mathrm{Na}{ }_{3} \mathrm{Cit}\right)$ which acts as both a reducing agent and a stabilizer is widely used for Au NPs synthesis (Turkevich method). ${ }^{3}$ For this purpose, a conventional heated batch reactor was often applied. ${ }^{4-9}$ In order for high yield synthesis of Au-NPs with well-defined shape and size, the Turkevich method has been extensively modified such as by changing the addition order of the reactants, using different reducing agents, adjusting the ratio of $\mathrm{HAuCl}_{4}$ to sodium citrate and $\mathrm{pH}$ values as well as adding new stabilisers. ${ }^{10-18}$ Replacement of water by its expensive isotope, deuterium oxide, was shown to produce smaller sizes of Au NPs $(5.3 \pm 1.1 \mathrm{~nm})$, compared with the particles $(9.0 \pm 1.2 \mathrm{~nm})$ produced in pure water which was attributed to faster reduction of precursor. ${ }^{19}$ An increase in reaction rate was observed by reversing the order of the reactants' addition (i.e. $\mathrm{HAuCl}_{4}$ to $\mathrm{Na}_{3} \mathrm{Cit}$ solution) that probably accelerated the thermal decomposition or oxidation of sodium citrate, resulting in the formation of Au-NPs in the 5-10 nm size range. ${ }^{11,12}$ Recently, synthesis of sub-10 nm citrate-stabilized Au NPs has been achieved following a kinetically controlled seeded-growth strategy, combining tannic acid with sodium citrate during nucleation. ${ }^{20}$ However, one of the major challenges in conventional batch systems has always been the non-uniform distribution of heat that results in temperature fluctuation, causing many variances in Au-NP synthesis processes, particularly in the nucleation and growth steps, ${ }^{21}$ resulting into wide particle size distribution. Furthermore the batch to batch difference also raise concerns about reproducibility and scaling-up issues. $^{21}$

The growth mechanism of the Turkevich method in a batch reactor and the influences of reagent ratio, reaction temperature and $\mathrm{pH}$ of the medium on synthesis of Au-NPs have recently been studied 
comprehensively in order to further control the nucleation and particle growth and then final particle size and size distribution. ${ }^{22}$ In this study, it was shown that the Turkevich synthesis followed a four-step seed-mediated growth mechanism in which no large particles $(>25 \mathrm{~nm})$ were formed during the synthesis, confirmed by in-situ ultrasmall angle X-ray scattering. ${ }^{22}$ In contrast to other proposed mechanisms, ${ }^{23}$ their observations excluded the formation of any large aggregates (e.g. $>25 \mathrm{~nm}$ ) throughout the Turkevich synthesis.

As an emerging heating technology, microwave heating $(\mathrm{MH})$ has been shown to dramatically reduce the reaction processing time and provide homogenous bulk heating to synthesise various distinctive nanocrystals with defined structures. Similar to the synthesis of other metal nanoparticles, ${ }^{7,8} \mathrm{MH}$ was employed in a batch reactor to prepare Au-NPs with various shapes including spherical, polygonal, rod, and triangular/prisms following the Turkevich or other synthesis routes using different reagents (e.g. 2,7-dihydroxy naphthalene as the reducing agent and cetyltrimethyl ammonium bromide as the stabilizer). ${ }^{16,24-27}$ These studies generally used domestic multimode microwave systems (600-1000 W) and their results revealed that microwave assisted fast temperature ramping was highly effective to synthesize Au-NPs (e.g. $20.01( \pm 2.05) \mathrm{nm}$ or $12.04( \pm 1.35) \mathrm{nm})$. Moreover, the results showed that the final products could vary in size and morphology depending on the type of reactor used, microwave absorption ability, reaction time etc. However, there is an obvious drawback in these reports that the particle size distribution and yield are not satisfactory due to the limited penetration depth of microwave irradiation $(\sim 1 \mathrm{~cm}$ in aqueous solution) and large reactor used (normally larger than $2 \mathrm{~cm}$ in diameter), which also affects scalability of the synthetic processes. ${ }^{7,8,28}$

It is well known that single mode microwave irradiation can transfer energy to the reaction medium much more efficiently and faster compared to multimode counterparts (e.g. domestic microwave systems). ${ }^{29,} 30$ Faster temperature ramping rates are likely to produce more seeds by promoting 
nucleation instead of particle growth, therefore smaller Au-NPs could be potentially achieved with a high yield by a single mode microwave irradiation even at very low microwave power. Furthermore, micro channel reactors were reported to have a better heat transfer rates compared with large diameter reactors due to the increased surface area-to-volume ratio. ${ }^{31}$ As such, one can anticipate that single mode microwave heating coupled with a micro-flow system which has a diameter smaller than the microwave penetration depth $(<1 \mathrm{~cm})$ should be potent to prepare Au-NPs with smaller particle size distribution and high yield. Given such an attractive approach, to the best of our knowledge, there is not a continuous microflow system driven by microwave irradiation reported to synthesise Au-NPs. Herein, a novel single mode microwave irradiation promoted microflow chemistry (SMMM) is presented to synthesise Au-NPs continuously with high reproducibility. Furthermore, the major variables e.g. microwave power, citrate-to-gold precursor molar ratio, residence time are comprehensively investigated and finally the SMMM is also compared with conventional flow system.

\section{Experimental}

The SMMM used consisted of a flow reactor $(6 \mathrm{~mL})$ made of $1 / 8^{\prime \prime}$ OD $(\sim 0.32 \mathrm{~cm})$ Teflon tubing, a commercially available single-mode microwave system (Discover, CEM Microwave Technology Ltd), a syringe pump (KD Scientific), a thermocouple temperature probe positioned $5 \mathrm{~cm}$ away from the exit of microwave heating zone, a sample and waste collection unit with backpressure regulator (20 psi, IDEX Health and Science) to control reactor pressure and an ice-bath placed between the end of the flow reactor and sample collection unit.

Two precursors hydrogen tetrachloroaurate (III) hydrate $\left(\mathrm{HAuCl}_{4} .3 \mathrm{H}_{2} \mathrm{O}, 99.9 \%\right)$ and trisodium citrate dehydrate $\left(\mathrm{Na}_{3} \mathrm{C}_{6} \mathrm{H}_{5} \mathrm{O}_{7}, 99 \%\right)$ were purchased from Sigma-Aldrich and used as received. In a typical protocol, $2 \mathrm{~mL}$ of freshly prepared solution of $\mathrm{HAuCl}_{4}(0.005 \mathrm{M})$ was added to a graduated cylinder and 
diluted to $25 \mathrm{~mL}$ using deionised water. The required amount of $\mathrm{Na}_{3} \mathrm{Cit}(0.05 \mathrm{M})$ to prepare the targeted citrate-to-gold molar ratio was then added to $\mathrm{HAuCl}_{4}$ aqueous solution, and the mixture of $\mathrm{HAuCl}_{4}$ and $\mathrm{Na}_{3} \mathrm{Cit}$ was diluted to $40 \mathrm{~mL}$ using deionised water to get $0.25 \mathrm{mM}$ of $\mathrm{HAuCl}_{4}$ concentration. This mixture of $\mathrm{HAuCl}_{4}$ and $\mathrm{Na}_{3} \mathrm{Cit}$ was immediately transferred into a $60 \mathrm{~mL}$ syringe. The mixture of $\mathrm{HAuCl}_{4}$ and $\mathrm{Na}_{3} \mathrm{Cit}$ then flowed through the reactor under a constant pressure. Residence times of approximately $0.6,0.85$ and $1.5 \mathrm{~min}$ that correspond to flow rates of 10,7 and $4 \mathrm{~mL} / \mathrm{min}$ were used, respectively. The hot fluid containing formed Au-NPs was immediately cooled by an ice-bath to quench any further reactions that may affect the homogeneity of Au-NPs product.

\section{Sample Characterization}

UV-vis analysis of Au-NPs was performed immediately after material synthesis and concurrently the samples for TEM analysis were prepared. Transmission electron microscopy (TEM) and high resolution transmission electron microscopy (HRTEM) were performed using Jeol JEM-1010 and JEOL-2010F coupled with an EDS detector (Oxford Instruments), respectively. TEM samples were prepared by dropping fresh solution of synthesized Au-NPs $(20 \mu \mathrm{L})$ on carbon coated TEM grids unless stated otherwise. Ultraviolet-visible (UV-Vis) absorption was recorded by Shimadzu UV-2550 UV-vis spectrophotometer. A typical Au-NP solution in de-ionized water $(\sim 3 \mathrm{~mL})$ was placed in a cell, and spectral analysis was performed in the range of $200-900 \mathrm{~nm}$. Size of Au-NPs was measured via ImageJ software, from their TEM images. The particle dimensions were measured in-plane, assuming that the width of the particles are comparable to their height. In case of non-spherical particle morphologies (e.g. boomerang and T-shape fused particles) observed, the longest particle dimensions either the length or width of the particle was measured to calculate particle aspect ratio. Descriptive statistical analysis was performed for individual data sets (Weight method: Direct weight, Variance divisor of moment: DF, 
Interpolation of Quantiles: Empirical distribution with averaging) using Origin 9.1 software to find out mean particle size and aspect ratio.

\section{Results and Discussion}

The SMMM was built up as depicted in Scheme $\mathbf{1}$ and used for continuous Au-NPs synthesis. Direct measurement of the temperature of hot fluid inside the micro-reactor is very challenging due to the size and configuration of the micro-reactor and small microwave cavity. Therefore, temperature of reaction fluid was recorded by using a thermocouple temperature probe placed inside T-type PTFE tubing and positioned $5 \mathrm{~cm}$ away from the exit of microwave irradiated zone. The temperature of the fluid in microwave zone is expected to be higher than that of the temperature recorded outside the microwave zone. The likely temperature in the microwave zone (just at the exit of the microwave irradiated zone) was estimated by considering forced convection heat transfer between the fluid inside the PTFE tubing and the outside surrounding air (see ESI for calculations). Heat loss due to radiation was neglected due to relatively low temperature of the fluid $\left(\sim 120^{\circ} \mathbf{C}\right)$. The calculated values of the fluid temperature at different fluid flow rates are listed in ESI Table S1.

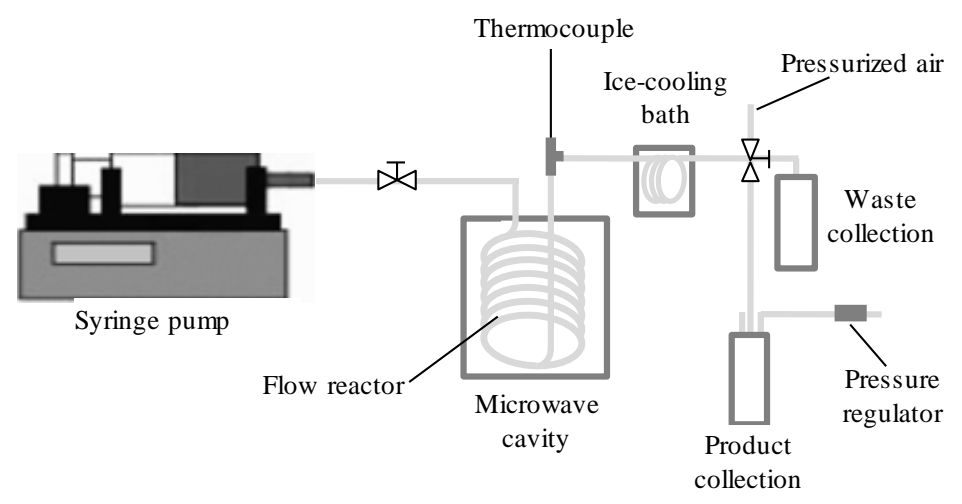

Scheme 1 Schematic representation of the combined single mode microwave irradiation and microflow technology to synthesize Au-NPs. 


\section{Influence of microwave irradiation intensity}

Formation of citrate stabilized Au-NPs was previously shown to follow a mechanism, including nucleation, aggregation, and spherical particle growth via Oswald ripening. ${ }^{14,22,32,33}$ For microwave assisted Au-NP synthesis, it is widely accepted that microwave irradiation accelerates the nucleation of particles due to its selective and fast heating characteristics. ${ }^{24-27,34}$ We have also recently found that a SMMM system has similar advantages including fast temperature ramping rate and selective heating of iron oxide for its self-assembly. ${ }^{30}$ Change in temperature ramp rates due to varied microwave powers is therefore expected to influence the nucleation rate of nanoparticles. Previous studies showed that AuNPs with uniform size $\sim 12.04 \pm 1.35 \mathrm{~nm}$ could be produced in $>5$ min after reaching $95{ }^{\circ} \mathrm{C}$ reaction temperature using microwave heating with fast ramping rate at $>50{ }^{\circ} \mathrm{C} \mathrm{min}^{-1}$ and maintaining the $\mathrm{pH}$ value as $>6.2$ by adjusting the $\mathrm{Na}_{3} \mathrm{Cit} / \mathrm{HAuCl}_{4}$ molar ratio $\sim 5.68 .{ }^{27}$ Based on these results, in order to examine the effect of microwave power, Au-NPs were synthesized at microwave powers of 20, 25, 30, 36, 40 and $45 \mathrm{~W}$, while maintaining citrate-to-gold molar ratio (6/1), flow rate $\left(4 \mathrm{~mL} \mathrm{~min}^{-1}\right)$ and back pressure (20 psi) constant. Temperature profiles of the aqueous solution were monitored for 5 min by measuring the temperature of the flowing reaction fluid outside the microwave zone at 30 second interval (see ESI Figure S1). Note that the volume of the reactor used was approximately $6 \mathrm{~mL}$, and the residence time of reaction fluid in the reactor was very short (e.g. $90 \mathrm{~s}$ at $4 \mathrm{~mL} / \mathrm{min}$ flow rate). In agreement with previous literature, ${ }^{27,29}$ temperature in SMMM system reached a certain value faster when higher microwave power was applied, compared to low microwave power (see ESI Figure S1). The temperature of the reaction fluid measured at the exit of microwave zone was $\sim 117{ }^{\circ} \mathrm{C}$ in approximately 2 min flow time and thereafter remained constant when microwave power was larger than $30 \mathrm{~W}$ (e.g. 36, 40 and $45 \mathrm{~W}$ ). On the other hand, temperature of the flowing reaction fluid did not stabilize when 20,25 or $30 \mathrm{~W}$ microwave power was applied. It showed a slightly increasing 
temperature trend between $2 \mathrm{~min}$ and $5 \mathrm{~min}$ (see ESI Figure S1). Clearly visible, coloured aqueous solutions of Au-NPs with increasing colour intensity were obtained in the case of 25, 30, 36, 40 and 45 W applied microwave power while the solution for $20 \mathrm{~W}$ applied microwave power was almost colourless, probably due to inadequate heating at low microwave power. Therefore in following studies $20 \mathrm{~W}$ microwave power was not considered and the sample prepared at this condition was not analysed. All coloured solutions were initially characterised by UV-vis absorption spectroscopy. Characteristic surface plasmon resonance (SPR) peaks of the obtained Au-NPs confirm continuous Au-NPs formation under 25, 30, 36, 40 and $45 \mathrm{~W}$ applied microwave power (Figure 1a). Au-NPs synthesized at microwave power from $25 \mathrm{~W}$ to $45 \mathrm{~W}$ display UV-vis absorption maxima positioned at approximately 560, 547, 542, 545 and $543 \mathrm{~nm}$, respectively (Figure 1a and c). Furthermore, a weak shoulder is observed at ca.750 $\mathrm{nm}$ for Au-NPs synthesized under $25 \mathrm{~W}$, that may probably be attributed to the presence of high aspect ratio (>2) Au-NPs observed in TEM images (ESI Figure S2). ${ }^{34,35}$ Typical TEM image of the Au-NPs synthesized under constant citrate-to-gold molar ratio of $6 / 1$ and $4 \mathrm{~mL} \mathrm{~min}^{-1}$ flow rate at $36 \mathrm{~W}$ is shown in Figure 1b. The high magnification HR-TEM image (inset of Figure 1b) proves $\mathrm{Au}-\mathrm{NPs}$ (average aspect ratio of 1.37) with lattice spacing consistent with $\mathrm{Au}^{0}(111)$ have been synthesised by the system. ${ }^{36}$

Careful inspection of the absorption spectra of Au-NPs reveals that the maximum absorption $\left(\lambda_{\max }\right)$ of Au-NPs synthesized at high microwave power (e.g. $45 \mathrm{~W}$ ) is blue-shifted compared to low microwave power (e.g. 25W) as shown in Figure 1c. For spherical Au-NPs, a blue-shift in $\lambda_{\max }$ is usually attributed to the formation of Au-NPs smaller in size. ${ }^{27,}{ }^{37}$ However, UV-vis spectroscopy alone is an inadequate technique for particle size and morphology analysis since the SPR of Au-NPs is highly morphology dependent, thus the use of alternative techniques such as electron microscopy is necessary to elucidate 
the reason of the observed $\lambda_{\max }$ shift. $^{9,26,27,34,35,38}$ Thus transmission electron microscopy (TEM) has been extensively used to observe the mean particle size and size distribution of Au-NPs. ${ }^{13,15,35}$
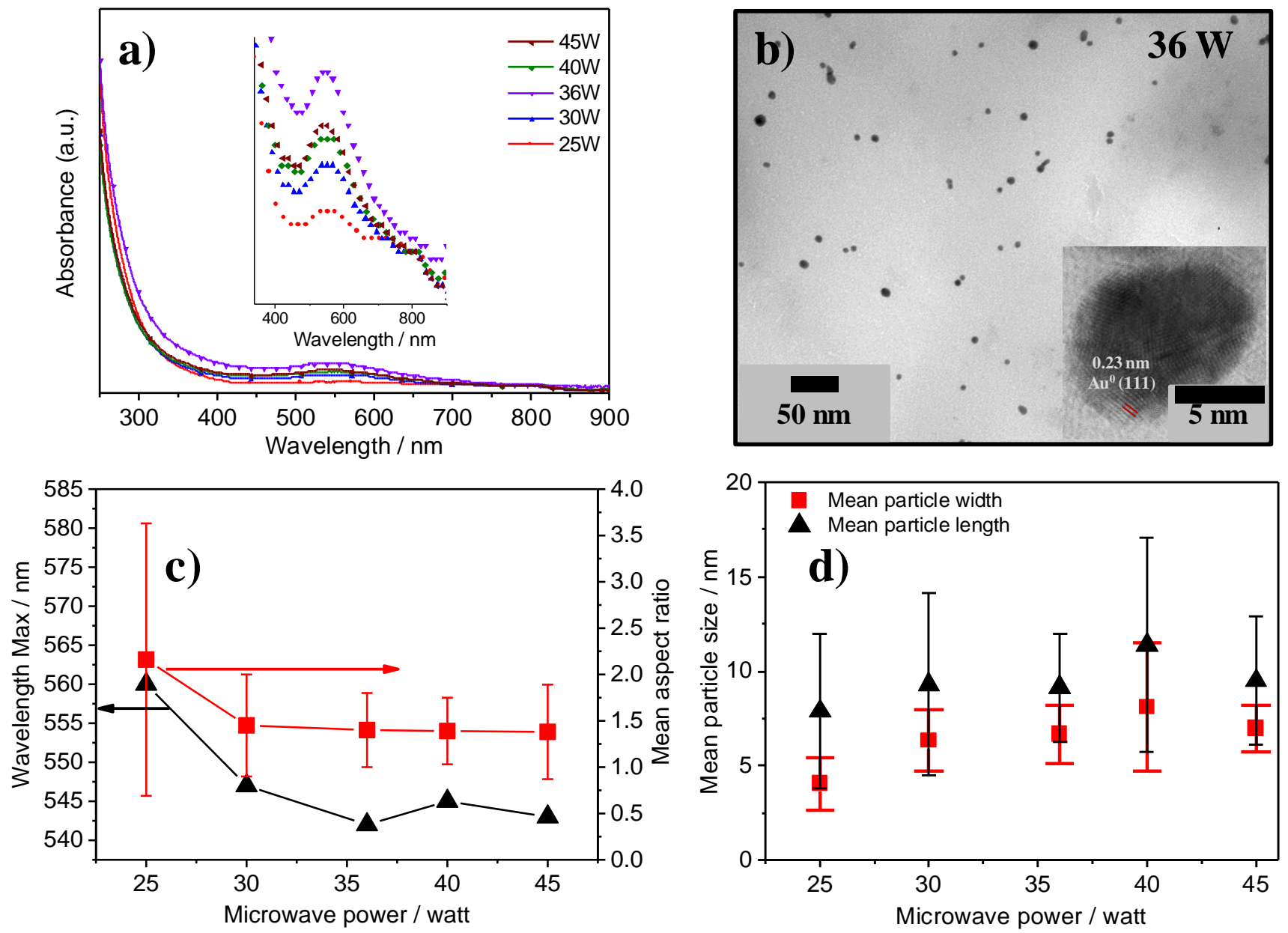

Figure 1 a) UV-vis spectra of the Au-NPs synthesised under the conditions given above. Inset: Enlarged UV-vis spectra of the Au-NPs synthesised. b) TEM images of the Au-NPs synthesized under constant citrate-to-gold molar ratio of $6 / 1$ and $4 \mathrm{~mL} \mathrm{~min}^{-1}$ flow rate by applying microwave power of $36 \mathrm{~W}$. Inset: High magnification HR-TEM image shows a selected non-spherical Au-NP (aspect ratio of 1.37) with lattice spacing $(0.23 \mathrm{~nm})$ consistent with $\mathrm{Au}^{0}(111)$. c) Graph shows the wavelength of maximum 
absorption $\left(\lambda_{\max }\right)$, the mean aspect ratio and the standard deviations in aspect ratio of the Au-NPs synthesized by applying microwave power of 25, 30, 36, 40 and 45 W. d) Graph shows the mean particle length/width and the standard deviations in size of the Au-NPs synthesized by applying microwave power of 25, 30, 36, 40 and $45 \mathrm{~W}$. Solid curves are intended to guide the eye.

TEM analysis of Au-NPs synthesized by the SMMM system shows the presence of a mixture of spherical and high-aspect ratio non-spherical Au-NPs, rather than commonly observed spherical Au-NPs formation via Turkevich method $^{12,22,39-41}$ (see ESI Figure S2 for TEM images). Further particle size and morphology were analysed by using corresponding TEM of the individual samples obtained from at least six different regions of TEM grid. The mean particle length, the mean particle width and the mean aspect ratio of Au-NPs and their standard deviations were then statistically obtained (Figure $1 \mathbf{c}$ and d), which is also summarized in ESI Table S2.

The maximum absorption $\left(\lambda_{\max }\right)$ is blue shifted when $25 \mathrm{~W}$ to $36 \mathrm{~W}$ of microwave power is applied. Thereafter the changes become small when microwave power further increases, while there is slightly red shift when $40 \mathrm{~W}$ of microwave power was applied (Figure 1c). Interestingly, the change of $\lambda_{\max }$ is relatively well correlated to the change of aspect ratio of the prepared Au-NPs at 25, 30, 36 and $45 \mathrm{~W}$ (Figure $1 \mathbf{b}$ and $\mathbf{c}$ ). Furthermore, the ratio of sphere-like particles to elongated particles were counted and shown in ESI Figure S3a. Notably there is lower percentage of spherical Au-NPs (40.5\%) prepared at $40 \mathrm{~W}$ which was derived using the amount of Au-NPs with aspect ratio smaller than $\sim 1.2$, compared to the Au-NPs prepared at $36(41.8 \%)$ and $45 \mathrm{~W}$ (47.7\%). In other words, there are more elongated particles when $40 \mathrm{~W}$ was applied, which causes the small red shift in the $\lambda_{\max }$ and also explain the larger mean particle length/width. 
Citrate stabilized spherical Au-NPs using conventional methods were previously reported by a few groups and, it was shown that the $\lambda_{\max }$ values of Au-NPs with particle size of 5-20 nm were in the range of 515-530 nm. ${ }^{15,39}$ In addition, microwave assisted batch synthesis of spherical Au-NPs with size distribution of $12.04 \pm 1.35 \mathrm{~nm}$ was reported, showing that smaller particles were produced at high microwave powers, attributed to higher supersaturation ratio of Au atoms in the solution at faster temperature ramp rates, ${ }^{27}$ which might be the case when $45 \mathrm{~W}$ microwave power was applied in this study. For spherical Au-NPs (aspect ratio 1), observed red shift in SPR peak position was generally attributed to change in the particle size or the variations in the refractive index of the surrounding medium of the nanoparticles. ${ }^{42}$ In contrast to previously reported citrate stabilized spherical Au-NPs (aspect ratio $\sim$ ) with $\lambda_{\max }$ at $c a .515-530 \mathrm{~nm},{ }^{15,27,39}$ the Au-NPs synthesized under 36, 40 and $45 \mathrm{~W}$ of microwave powers in a SMMM system show $\lambda_{\max }$ at $c a .542-545 \mathrm{~nm}$ although their size is below $10 \mathrm{~nm}$ (ESI Table S2). Therefore large $\lambda_{\max }$ for Au-NPs produced in the SMMM system is attributed to the relatively large aspect ratios in the current study (Figure 1c and ESI Table S2).

\section{Influence of citrate-to-gold molar ratio $([\mathrm{Cit}] /[\mathrm{Au}])$}

Given that the relatively high intensity UV-vis absorption of Au-NPs achieved at microwave power of $36 \mathrm{~W}$ indicated by its UV-vis intensity, Au-NPs were further synthesized continuously by varying $[\mathrm{Cit}] /[\mathrm{Au}]$ in the range 1-60 at fixed microwave power of $36 \mathrm{~W}$ and $4 \mathrm{~mL} \min ^{-1}$ flow rate. The temperature just outside the microwave zone increased to $\sim 118-119{ }^{\circ} \mathrm{C}$ in approximately 2 min fluid flow for all $[\mathrm{Cit}] /[\mathrm{Au}]$ ratios used (ESI Figure S4). Aqueous solutions of Au-NPs were collected after the outside temperature remained at $\sim 118-119{ }^{\circ} \mathrm{C}$ (after $2 \mathrm{~min}$ ). Au-nanowire-like structures with polygonal heads (width $\times$ length: $\sim 10 \times \geq 200 \mathrm{~nm}$ ) were obtained in a short time $(2 \mathrm{~min})$ when $[\mathrm{Cit}] /[\mathrm{Au}]$ of 1/1 is used (Figure 2), probably due to preferential adsorption of $\mathrm{AuCl}_{4}{ }^{-}$on the surface of the initially 
formed thermodynamically unstable gold nanoparticles. This process is favourable when the stabiliser citrate ions are not enough to fully cover their surface.

${ }^{33}$ Similar gold nanowire formation at $80{ }^{\circ} \mathrm{C}$ in 30 min was observed in a batch reactor by citrate reduction of $\mathrm{AuCl}_{4}{ }^{-}$with a low concentration of citrate ${ }^{33}$ although it has recently been reported that no particles longer that $25 \mathrm{~nm}$ have been observed during Au-NPs synthesis using the Turkevich method. ${ }^{22}$

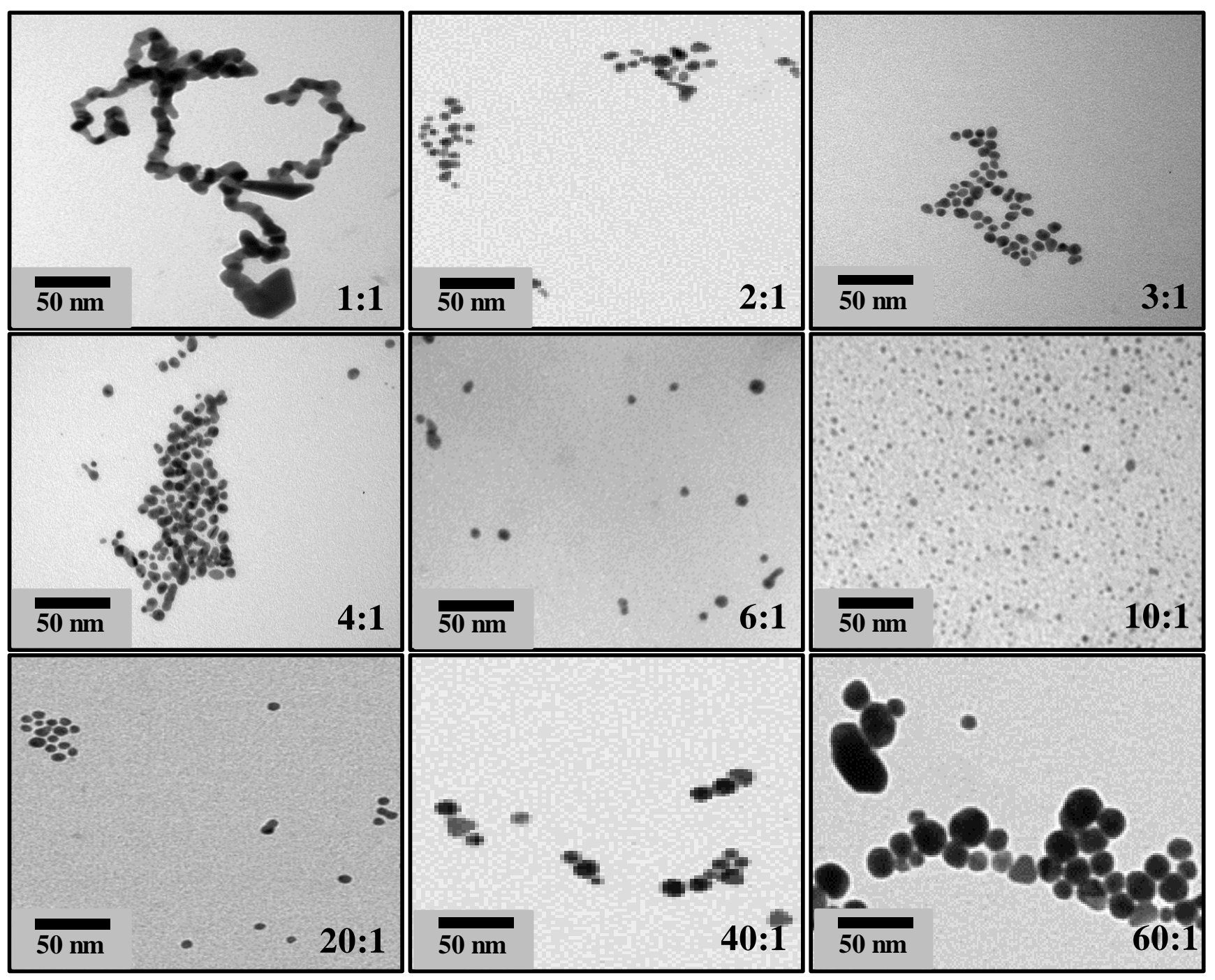

Figure 2 Typical TEM images of the Au-NPs synthesized under constant microwave power of $36 \mathrm{~W}, 4$ $\mathrm{mL} \min ^{-1}$ flow rate by using citrate-to-gold molar ratios of $1,2,3,4,6,10,20,40$ and 60 . 


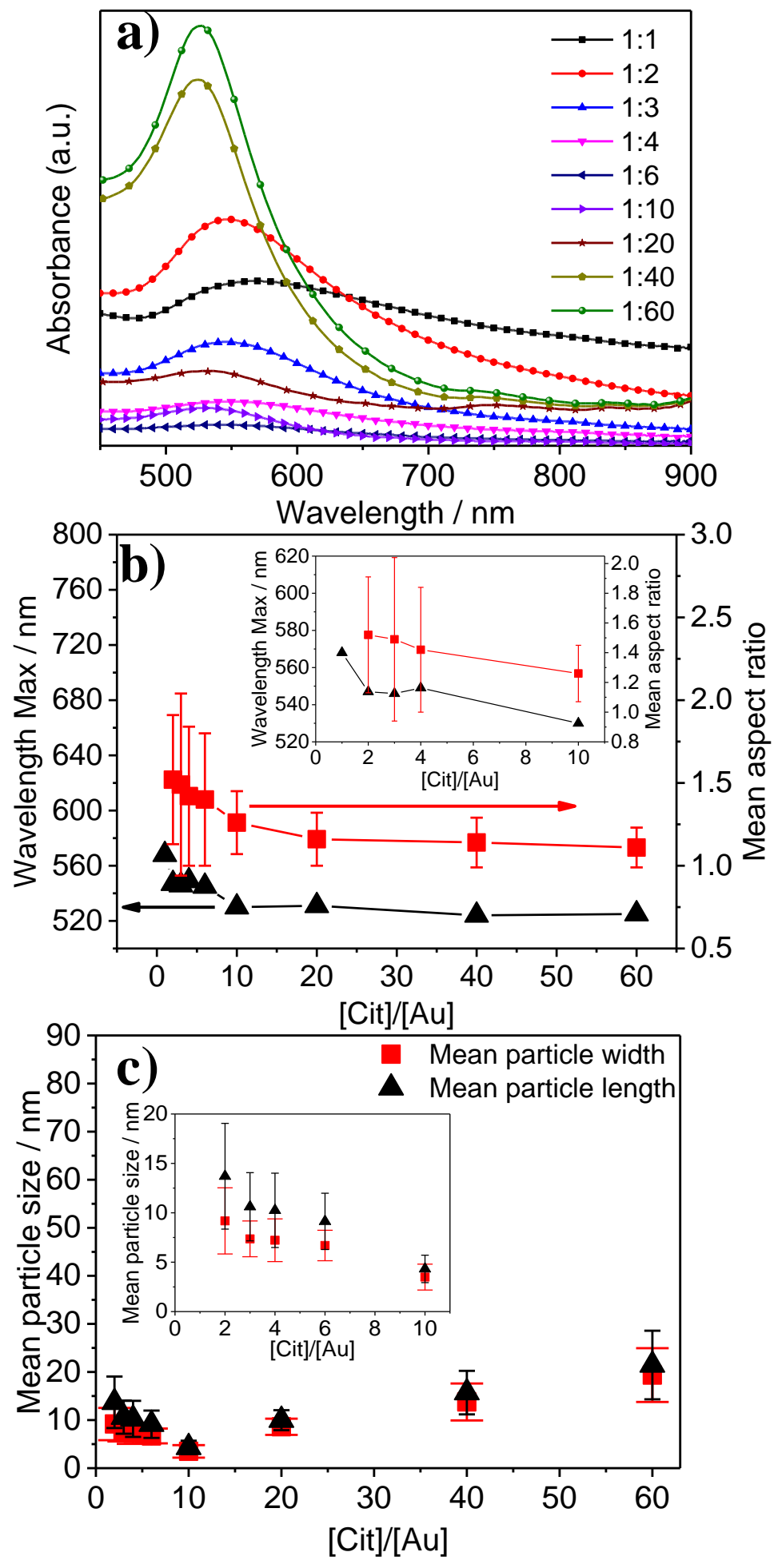

Page 13 of 25 
Figure 3 a) UV-vis spectra of the Au-NPs synthesized under constant microwave power of $36 \mathrm{~W}, 4 \mathrm{~mL}$ $\mathrm{min}^{-1}$ flow rate and 20 psi back pressure by using citrate-to-gold molar ratios of 1, 2, 3, 4, 6, 10, 20, 40 and 60. b) Graph shows the wavelength of maximum absorption $\left(\lambda_{\max }\right)$ and the mean aspect ratio and the standard deviations in aspect ratio of the Au-NPs synthesized by using [Cit]/[ $\mathrm{Au}]$ of 2, 3, 4, 6, 10, 20, 40 and 60. Note that, for $[\mathrm{Cit}] /[\mathrm{Au}]$ of $1 / 1$, only $\lambda_{\max }$ absorption was presented since aspect ratio of gold-nanowires was not calculated. Inset shows the region from [Cit]/[ $\mathrm{Au}]$ of 2 to 10 . c) Graph shows the mean particle length/width and the standard deviations in size of the Au-NPs synthesized by using $[\mathrm{Cit}] /[\mathrm{Au}]$ of $2,3,4,6,10,20,40$ and 60 . Note that there is no data point for $[\mathrm{Cit}] /[\mathrm{Au}]$ of $1 / 1$. Inset shows the region from $[\mathrm{Cit}] /[\mathrm{Au}]$ of 2 to 10 . Solid curves are intended to guide the eye.

After the TEM observation, UV-vis analysis of produced Au-NPs were carried out. It shows $\lambda_{\max }$ at $c a$. $547,546,549,545,530,531,524$ and $525 \mathrm{~nm}$ for $[\mathrm{Cit}] /[\mathrm{Au}]$ ratios of 2, 3, 4, 6, 10, 20, 40 and 60, respectively (Figure 3 a and b). Consistent with the literature, ${ }^{35,43}$ as-synthesized Au-nanowires using $[\mathrm{Cit}] /[\mathrm{Au}]$ of $1 / 1$ show a broad UV-vis absorption band $\left(\lambda_{\max } \sim 568 \mathrm{~nm}\right)$ with a long tail in near IR region, characteristic of Au-nanowires (Figure $3 \mathbf{a}$ and b). It is known that high aspect ratio elongated Au-NPs possess both SPR band positioned at ca. $520 \mathrm{~nm}$ (so-called transverse band), characteristic of spherical Au-NPs, and another absorption band, so-called longitudinal band, at relatively longer wavelengths owing to the plasmon oscillation of electrons along the long axis of the elongated Au-NPs. ${ }^{35,42}$ These bands are clearly separated from each other for the high aspect ratio well-defined Au-NPs. In addition, they may significantly get closer in some circumstances and may appear as a broadened single band for the low aspect ratio (or spherical) Au-NPs. ${ }^{35}$ However, in our study formed gold nanowires show a board peak observed from $500 \mathrm{~nm}$ to $900 \mathrm{~nm}$ with a significantly flattened absorption pattern after 800 
$\mathrm{nm}$. Consistent with the previous report, this broad profile may be attributed to the superposition of the longitudinal resonance of gold nanowires with varying lengths at this wavelength range. ${ }^{33}$

The statistically derived mean particle size of the produced Au-NPs decreases when [Cit]/[Au] ratios increase from $2 / 1$ to $10 / 1$ and reaches its minimum at the ratio of 10/1 (Figure 3c). After that, the mean particle size of the produced Au-NPs shows an increasing trend. Thus the smallest (mean particle length $=4.3 \mathrm{~nm}$; mean particle width=3.5 nm) and the largest (mean particle length=21.5 nm; mean particle width=19.4 nm) Au-NPs are produced when [Cit]/[Au]s of 10/1 and 60/1 are used, respectively (see ESI Table S3), which is agreement with Au NPs prepared in a batch reactor by conventional heating ${ }^{14,44}$ Furthermore, a clear dependency of particle size of produced Au-NPs on citrate ratio was observed at constant $\mathrm{HAuCl}_{4}$ precursor concentration $(0.25 \mathrm{mM})$ in the SMMM system. In other words, the concentration of citrate has non-monotonic effect on the final size of the produced Au-NPs, as reported by Kumar et $a l .{ }^{45}$ and Polte et $a l .{ }^{22}$. It is proposed that the change in particle size with citrate concentration is probably due to the capping effect of the citrate ions. ${ }^{27}$ More citrate ions, better capping effect, leading to smaller particles. Further increasing citrate ions concentration, reduction of gold precursor is dominated which potentially may accelerate particle growth. The detailed reason is currently underway.

One can observe there is a correlation between the particle size and $\lambda_{\max }$ absorption (Figure $\mathbf{3 b}$ and $\mathbf{c}$ ). The mean aspect ratio of the produced $\mathrm{Au}-\mathrm{NPs}$ shows a decreasing trend when $[\mathrm{Cit}] /[\mathrm{Au}]$ ratios increase from 2/1 to 20/1. Thereafter, it remains relatively unchanged. Again a correlation between the particle aspect ratio and $\lambda_{\max }$ absorption is observed, the higher the aspect ratio the larger the $\lambda_{\max }$, (Figure $\mathbf{3 b}$ ). In particular, this correlation is observed when two materials prepared under different condition but with similar mean particle lengths. For instance, the Au NPs prepared at [Cit]/[Au] ratios of 4/1 and 20/1 show mean particle length of 10.3 and $9.9 \mathrm{~nm}$. (see ESI Table S3). Although the mean particle lengths 
are comparable, their $\lambda_{\max }$ absorptions are observed at 549 and $531 \mathrm{~nm}$, respectively, corresponding to mean aspect ratio of 1.42 and 1.16 (Figure $3 \mathbf{b}$ ). Therefore we would like to propose that the aspect ratio dominates to some extend to the $\lambda_{\max }$ absorption of the Au-NPs synthesized by the SMMM system. A previous study showed the effect of the Au-NPs geometry on the localized surface plasmons (LSPs) theoretically and experimentally. By comparing large oblate, prolate and ellipsoid shaped Au-NPs (>50 $\mathrm{nm})^{46}$ the study indicated that the LSPs were highly sensitive to change in the dimensions of ellipsoid particles, suggesting a correlation between the aspect ratio and the $\lambda_{\max }$ absorption of the Au-NPs. It was also reported that the overlapped particles (or junctions), so-called hot-spots occurring between a pair of adjacent nanoparticles, could result in highly intense and localized electromagnetic fields upon excitation by incident light of the appropriate polarization. The region of the overlap between particles

or the distance between two adjacent nanoparticles was responsible for the shift of the SPR peak. ${ }^{47-51}$ In agreement with these studies, close inspections on HRTEM images of the Au-NPs produced by the SMMM system clearly reveal the formation of interconnected particles (see ESI Figure 5). The interconnection would alter the size of the resulting Au-NPs, which is probably responsible for increasing of the aspect ratio and the observed red-shift in the $\lambda_{\max }$ absorption of the Au-NPs. It is also worth mentioning that the produced largest Au-NPs produced $(21.5 \pm 7.1 \mathrm{~nm} / 19.4 \pm 5.6 \mathrm{~nm}$; length/width) prepared using [Cit]/[Au] ratios of 60/1 show relatively blue-shifted $\lambda_{\max }$ absorption compared to AuNPs produced using other $[\mathrm{Cit}] /[\mathrm{Au}]$ ratios, again likely due to the amount of the highest percentage of Au-NPs with aspect ratio smaller than $\sim 1.2$, which is $90 \%$ (see ESI Table S3 and Figure S3b).

\section{Influence of flow rate}

Furthermore we studied the influence of reactant flow rates on the produced Au-NPs when other reaction parameters (reactor volume $6 \mathrm{~mL}$, microwave power $36 \mathrm{~W}$ and applied back pressure 20 psi) 
were kept constant. The citrate-to-gold ratio of 60/1 was used since it produced Au-NPs with the highest UV-vis absorption intensity at $525 \mathrm{~nm}$ (see Figure 3a), and the reaction was carried out at flow rates of 4, 7 and $10 \mathrm{~mL} \mathrm{~min} \min ^{-1}$. $4 \mathrm{mLin}^{-1}$ flow rate was found as the reliable flow rate in the current configuration (see ESI Figure S6a-d). A clear red solution confirming Au-NP formation was obtained at flow rate of $4 \mathrm{~mL} \mathrm{~min}^{-1}$, compared to 7 and $10 \mathrm{~mL} \mathrm{~min}^{-1}$ which yielded a cloudy grey solution (see photographs in Figure S6a-c). TEM image of the red solution displays Au-NPs with mean length/width size of $21.5 \pm 7.1 \mathrm{~nm} / 19.4 \pm 5.6 \mathrm{~nm}$ (Figure S6a). In contrast, TEM analysis of the solutions obtained at flow rate of 7 and $10 \mathrm{~mL} \mathrm{~min}^{-1}$ shows a mixture of Au-NPs formed of spherical, high aspect ratio and rod-like particles (Figure S6b and $\mathbf{6 c}$ ). Electron microscopy results reveal that flow rate is critical to produce Au-NPs with well-defined morphology under given experimental conditions. Complementary UV-vis spectroscopy further supports the formation of Au-NPs at $4 \mathrm{~mL} \mathrm{~min}^{-1}$ flow rate, showing a strong SPR band at $524 \mathrm{~nm}$, compared to a weak one at $575 \mathrm{~nm}$ for $7 \mathrm{~mL} \mathrm{~min}^{-1}$ flow rate (Figure S6d). However, no clearly visible SPR band is observed in the enlarged UV-vis spectrum (Figure S6d) for 10 $\mathrm{mL} \mathrm{min}^{-1}$ flow rate although TEM analysis showed some particles' formation (Figure S6c), probably due to very low concentration of Au-NP at such a short reaction time.

\section{Reproducibility of synthesis}

Reproducibility of the SMMM system was tested by repeating the fabrication of Au-NPs three times under microwave power of $36 \mathrm{~W}, 4 \mathrm{~mL} \mathrm{~min}^{-1}$ flow rate and 20 psi back pressure by using citrate-to-gold molar ratios of 6/1. Au-NPs were characterized by both UV-vis spectroscopy and TEM. In all repeated experiments UV-vis analysis of produced particles shows absorption maxima at ca. $542 \mathrm{~nm}$ (Figure 4a) and, TEM analysis indicates Au-NPs with particle size distribution of $\sim 10 \mathrm{~nm}$ (length) and $\sim 7 \mathrm{~nm}$ (width) (Figure 4b-c), confirming that the novel synthesis method is reproducible and capable of producing AuNPs with similar size distributions, which is extremely important for nanomaterial manufacturing 

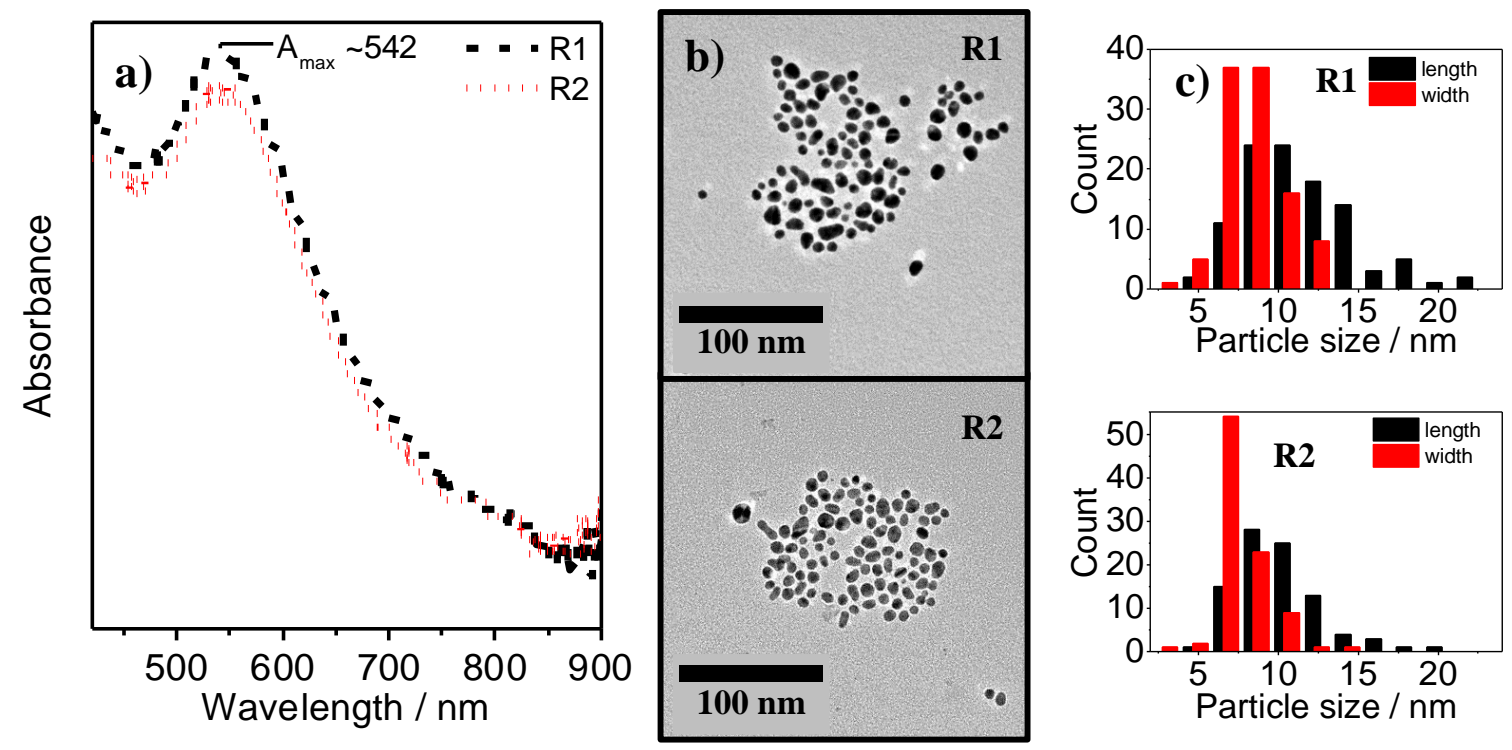

Figure 4 a) UV-vis spectra of the Au-NPs synthesized under constant microwave power of $36 \mathrm{~W}, 4 \mathrm{~mL}$ $\min ^{-1}$ flow rate by using citrate-to-gold molar ratios of 6/1. b) TEM images of Au-NPs produced by two different repeat experiments (R1 and R2) under the same experimental conditions and c) the particle size distribution of the produced Au-NPs.

On the other hand, Au-NPs prepared by conventional heating at $100{ }^{\circ} \mathrm{C}$ under identical experimental conditions (citrate-to-gold ratio of $6 / 1,4 \mathrm{~mL} \mathrm{~min}^{-1}$ ) show mean length/width size of $48.7( \pm 18.6) \mathrm{nm} / 35.9( \pm 10.1) \mathrm{nm}$, suggesting the superiority of microwave assisted fast nucleation in a flow system over conventional heating (Figure 5).
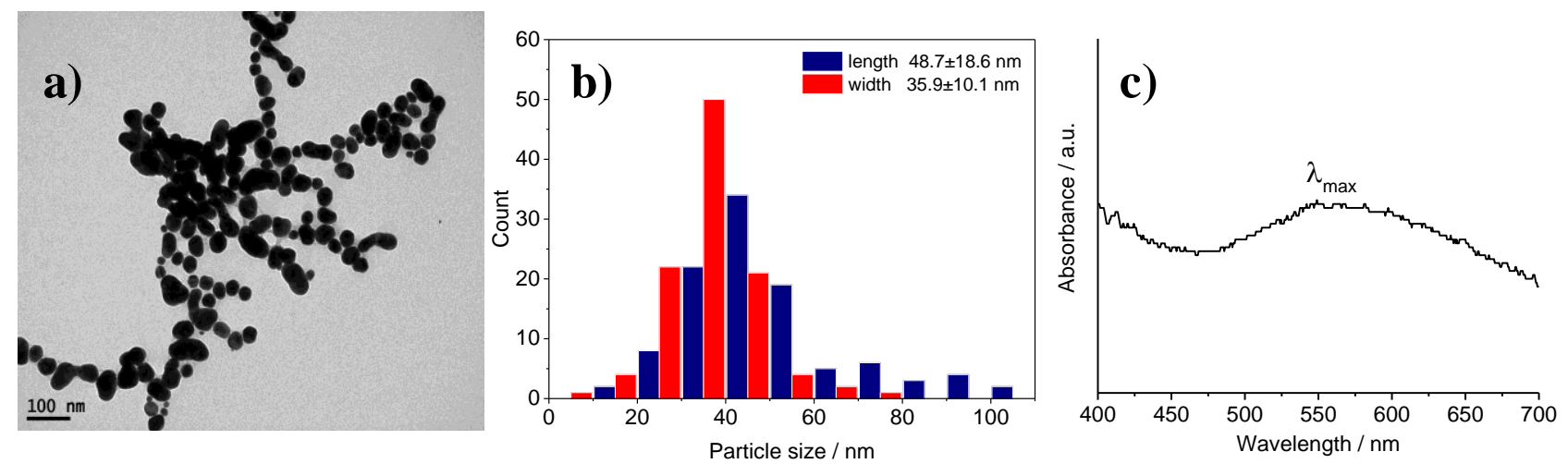

Page 18 of 25 
Figure 5 a) TEM images of the Au-NPs synthesized at constant citrate-to-gold molar ratio of 6/1, $4 \mathrm{~mL} \mathrm{~min}^{-1}$ flow rate and 20 psi back pressure by conventional heating at $100{ }^{\circ} \mathrm{C}$. b) The particle size distribution of the produced Au-NPs. c) UV-spectrum of the produced Au-NPs. $\lambda_{\max }$ is observed at $c a .550 \mathrm{~nm}$.

\section{Conclusion}

For the first time, a combining single mode microwave irradiation and microflow system (SMMM) has successfully been used to synthesize Au-NPs with controlled particle size (mean particle width $\times$ length in the range of $\sim 4 \times 8 \mathrm{~nm}$ and $\sim 8 \times 12 \mathrm{~nm}$ ) and mean aspect ratio (between $\sim 1.4$ and 2.2) with just $90 \mathrm{~s}$ reaction time. Au-nanowire-like structure with polygonal heads (width $\times$ length: $\sim 10 \times \geq 200 \mathrm{~nm}$ ) is produced at the citrate-to-gold ratio of $1 / 1$. Increasing the citrate-to-gold ratio from $2 / 1$ to $10 / 1$ leads to a decrease in particle size, however further increase from 10/1 to 60/1 results in an increase in particle size which is likely due to dual function of citrate ions. Interestingly, Au-NPs smaller than $15 \mathrm{~nm}$ with relatively higher mean aspect ratios (> 1.4) show red-shifted SPR peaks (>540 $\mathrm{nm})$ compared to those $(<530 \mathrm{~nm})$ previously reported with similar particle size of Au-NPs but mean aspect ratios closer to $\sim 1$, suggesting the strong dependency of SPR peak on the aspect ratio of produced Au-NPs, which is further witnessed by the correlation of the maximum absorption $\left(\lambda_{\max }\right)$ with aspect ratio achieved in three different conditions, changing microwave power, precursor ratios and flow rates. It is also proposed that the change in the particle aspect ratio is related to the formation of particle-particle junctions (observed by HRTEM), which is expected to have an influence on both the particle size and the aspect ratio. In addition, the novel synthesis approach has been proved reliable and reproducible.

In brief, the developed SMMM system combining the advantages of both single mode microwave heating and flow chemistry allows to control the reaction variables (e.g. heat, temperature, cooling, reactant concentration, time, reaction volume etc.) immediately and effectively during the course of the reaction, resulting into much smaller particle size and size distribution and at the same time benefiting from 
fast and high throughput synthesis with good reproducibility between runs. It has also a strong potential to continuously fabricate metal and metal oxide nanoparticles in an economical manner.

\section{Supporting Information}

Fluid temperatures outside the microwave heating zone, tables of mean particle length and width and mean aspect ratio of the produced Au-NPs by using different microwave powers or citrate-to-gold molar ratios, TEM images of these particles synthesized at different microwave powers or flow rates, and UV-vis spectra of the Au-NPs synthesized at varying fluid flow rates.

\section{Acknowledgements}

All authors acknowledge the Leverhulme Trust (RPG-2012-582) for financial support.

\section{References}

1. Huang, X.; Jain, P. K.; El-Sayed, I. H.; El-Sayed, M. A., Gold nanoparticles: interesting optical properties and recent applications in cancer diagnostics and therapy. Nanomedicine 2007, 2, (5), 681693.

2. D. Howes, P.; Rana, S.; M. Stevens, M., Plasmonic nanomaterials for biodiagnostics. Chemical Society Reviews 2014, 43, (11), 3835-3853.

3. Turkevich, J.; Stevenson, P. C.; Hillier, J., A study of the nucleation and growth processes in the synthesis of colloidal gold. Discussions of the Faraday Society 1951, 11, (0), 55-75.

4. Sebastian Cabeza, V.; Kuhn, S.; Kulkarni, A. A.; Jensen, K. F., Size-Controlled Flow Synthesis of Gold Nanoparticles Using a Segmented Flow Microfluidic Platform. Langmuir 2012, 28, (17), 70077013.

5. Polte, J.; Herder, M.; Erler, R.; Rolf, S.; Fischer, A.; Wurth, C.; Thunemann, A. F.; Kraehnert, R.; Emmerling, F., Mechanistic insights into seeded growth processes of gold nanoparticles. Nanoscale 2010, 2, (11), 2463-2469. 
6. Polte, J.; Ahner, T. T.; Delissen, F.; Sokolov, S.; Emmerling, F.; Thünemann, A. F.; Kraehnert, R., Mechanism of Gold Nanoparticle Formation in the Classical Citrate Synthesis Method Derived from Coupled In Situ XANES and SAXS Evaluation. Journal of the American Chemical Society 2010, 132, (4), 1296-1301.

7. Baghbanzadeh, M.; Carbone, L.; Cozzoli, P. D.; Kappe, C. O., Microwave-Assisted Synthesis of Colloidal Inorganic Nanocrystals. Angewandte Chemie International Edition 2011, 50, (48), 1131211359.

8. Bilecka, I.; Niederberger, M., Microwave chemistry for inorganic nanomaterials synthesis. Nanoscale 2010, 2, (8), 1358-1374.

9. Zhu, Y.-J.; Chen, F., Microwave-Assisted Preparation of Inorganic Nanostructures in Liquid Phase. Chemical Reviews 2014, 114, (12), 6462-6555.

10. Turkevich, J.; Stevenson, P. C.; Hillier, J., The Formation of Colloidal Gold. The Journal of Physical Chemistry 1953, 57, (7), 670-673.

11. Ojea-Jiménez, I.; Bastús, N. G.; Puntes, V., Influence of the Sequence of the Reagents Addition in the Citrate-Mediated Synthesis of Gold Nanoparticles. The Journal of Physical Chemistry C 2011, $115,(32), 15752-15757$.

12. Sivaraman, S. K.; Kumar, S.; Santhanam, V., Monodisperse sub-10\&\#xa0;nm gold nanoparticles by reversing the order of addition in Turkevich method - The role of chloroauric acid. Journal of Colloid and Interface Science 2011, 361, (2), 543-547.

13. Ftouni, J.; Penhoat, M.; Addad, A.; Payen, E.; Rolando, C.; Girardon, J.-S., Highly controlled synthesis of nanometric gold particles by citrate reduction using the short mixing, heating and quenching times achievable in a microfluidic device. Nanoscale 2012, 4, (15), 4450-4454.

14. Ji, X.; Song, X.; Li, J.; Bai, Y.; Yang, W.; Peng, X., Size control of gold nanocrystals in citrate reduction: The third role of citrate. J Am Chem Soc 2007, 129, 13939.

15. Li, C.; Li, D.; Wan, G.; Xu, J.; Hou, W., Facile synthesis of concentrated gold nanoparticles with low size-distribution in water: temperature and pH controls. Nanoscale Research Letters 2011, 6, (1), 440.

16. Ngo, V. K. T.; Nguyen, H. P. U.; Huynh, T. P.; Tran, N. N. P.; Lam, Q. V.; Huynh, T. D., Preparation of gold nanoparticles by microwave heating and application of spectroscopy to study conjugate of gold nanoparticles with antibody E. coli O157:H7. Advances in Natural Sciences: Nanoscience and Nanotechnology 2015, 6, (3), 035015. 
17. Nguyen, D.; Kim, D.; So, M.; Kim, K., Experimental measurements of gold nanoparticle nucleation and growth by citrate reduction of HAuCl4. Adv Powder Technol 2010, 21, 111.

18. Volkert, A.; Subramaniam, V.; Haes, A., Implications of citrate concentration during the seeded growth synthesis of gold nanoparticles. Chem Commun 2011, 47, 478.

19. Ojea-Jimenez, I.; Romero, F.; Bastus, N.; Puntes, V., Small Gold Nanoparticles Synthesized with Sodium Citrate and Heavy Water: Insights into the Reaction Mechanism. J Phys Chem C 2010, 114, 1800.

20. Piella, J.; Bastús, N. G.; Puntes, V., Size-Controlled Synthesis of Sub-10-nanometer CitrateStabilized Gold Nanoparticles and Related Optical Properties. Chemistry of Materials 2016, 28, (4), 1066-1075.

21. Abou-Hassan, A.; Sandre, O.; Cabuil, V., Microfluidics in Inorganic Chemistry. Angewandte Chemie International Edition 2010, 49, (36), 6268-6286.

22. Wuithschick, M.; Birnbaum, A.; Witte, S.; Sztucki, M.; Vainio, U.; Pinna, N.; Rademann, K.; Emmerling, F.; Kraehnert, R.; Polte, J., Turkevich in New Robes: Key Questions Answered for the Most Common Gold Nanoparticle Synthesis. ACS Nano 2015, 9, (7), 7052-7071.

23. Pong, B.-K.; Elim, H. I.; Chong, J.-X.; Ji, W.; Trout, B. L.; Lee, J.-Y., New Insights on the Nanoparticle Growth Mechanism in the Citrate Reduction of Gold(III) Salt: Formation of the Au Nanowire Intermediate and Its Nonlinear Optical Properties. The Journal of Physical Chemistry C 2007, 111, (17), 6281-6287.

24. Kundu, S.; Peng, L.; Liang, H., A New Route to Obtain High-Yield Multiple-Shaped Gold Nanoparticles in Aqueous Solution using Microwave Irradiation. Inorganic Chemistry 2008, 47, (14), 6344-6352.

25. Liu, F.-K.; Ker, C.-J.; Chang, Y.-C.; Ko, F.-H.; Chu, T.-C.; Dai, B.-T., Microwave Heating for the Preparation of Nanometer Gold Particles. Japanese Journal of Applied Physics 2003, 42, (6S), 4152. 26. Seol, S. K.; Kim, D.; Jung, S.; Chang, W. S.; Bae, Y. M.; Lee, K. H.; Hwu, Y., Effect of citrate on poly(vinyl pyrrolidone)-stabilized gold nanoparticles formed by PVP reduction in microwave (MW) synthesis. Materials Chemistry and Physics 2012, 137, (1), 135-139.

27. Seol, S. K.; Kim, D.; Jung, S.; Hwu, Y., Microwave synthesis of gold nanoparticles: Effect of applied microwave power and solution pH. Materials Chemistry and Physics 2011, 131, (1-2), 331-335. 
28. Mohamed, M. B.; AbouZeid, K. M.; Abdelsayed, V.; Aljarash, A. A.; El-Shall, M. S., Growth Mechanism of Anisotropic Gold Nanocrystals via Microwave Synthesis: Formation of Dioleamide by Gold Nanocatalysis. ACS Nano 2010, 4, (5), 2766-2772.

29. Sturm, G. S. J.; Verweij, M. D.; van Gerven, T.; Stankiewicz, A. I.; Stefanidis, G. D., On the effect of resonant microwave fields on temperature distribution in time and space. International Journal of Heat and Mass Transfer 2012, 55, (13-14), 3800-3811.

30. Bayazit, M. K.; Cao, E.; Gavriilidis, A.; Tang, J., A microwave promoted continuous flow approach to self-assembled hierarchical hematite superstructures. Green Chemistry 2016, 18, (10), 30573065.

31. Horikoshi, S.; Abe, H.; Torigoe, K.; Abe, M.; Serpone, N., Access to small size distributions of nanoparticles by microwave-assisted synthesis. Formation of $\mathrm{Ag}$ nanoparticles in aqueous carboxymethylcellulose solutions in batch and continuous-flow reactors. Nanoscale 2010, 2, (8), 14411447.

32. Pong, B.; Elim, H.; Chong, J.; Ji, W.; Trout, B.; Lee, J., New insights on the nanoparticle growth mechanism in the citrate reduction of Gold(III) salt: Formation of the au nanowire intermediate and its nonlinear optical properties. J Phys Chem C 2007, 111, 6281.

33. Pei, L.; Mori, K.; Adachi, M., Formation Process of Two-Dimensional Networked Gold Nanowires by Citrate Reduction of AuCl4- and the Shape Stabilization. Langmuir 2004, 20, (18), 78377843.

34. Saverot, S.-E.; Reese, L. M.; Cimini, D.; Vikesland, P. J.; Bickford, L. R., Characterization of Conventional One-Step Sodium Thiosulfate Facilitated Gold Nanoparticle Synthesis. Nanoscale research letters 2015, 10, (1), 940-940.

35. Omi, H., Determination of Aspect-Ratio Distribution in Gold Nanowires Using Absorption Spectra and Transmission Electron Microscopy Techniques. INTECH Open Access Publisher: 2012.

36. Bayazit, M. K.; Hodge, S. A.; Clancy, A. J.; Menzel, R.; Chen, S.; Shaffer, M. S. P., Carbon nanotube anions for the preparation of gold nanoparticle-nanocarbon hybrids. Chemical Communications 2016, 52, (9), 1934-1937.

37. Haiss, W.; Thanh, N. T. K.; Aveyard, J.; Fernig, D. G., Determination of Size and Concentration of Gold Nanoparticles from UV-Vis Spectra. Analytical Chemistry 2007, 79, (11), 4215-4221. 
38. Gutierrez-Wing, C.; Esparza, R.; Vargas-Hernandez, C.; Fernandez Garcia, M. E.; JoseYacaman, M., Microwave-assisted synthesis of gold nanoparticles self-assembled into self-supported superstructures. Nanoscale 2012, 4, (7), 2281-2287.

39. Kimling, J.; Maier, M.; Okenve, B.; Kotaidis, V.; Ballot, H.; Plech, A., Turkevich Method for Gold Nanoparticle Synthesis Revisited. The Journal of Physical Chemistry B 2006, 110, (32), 1570015707.

40. Uppal, M.; Kafizas, A.; Ewing, M.; Parkin, I., The effect of initiation method on the size, monodispersity and shape of gold nanoparticles formed by the Turkevich method. New J Chem 2010, 34, 2906.

41. Uppal, M.; Kafizas, A.; Lim, T.; Parkin, I., The extended time evolution size decrease of gold nanoparticles formed by the Turkevich method. New J Chem 2010, 34, 1401.

42. Chen, H.; Kou, X.; Yang, Z.; Ni, W.; Wang, J., Shape- and Size-Dependent Refractive Index Sensitivity of Gold Nanoparticles. Langmuir 2008, 24, (10), 5233-5237.

43. Shankar, R.; Chaudhary, M.; Molloy, K. C.; Kociok-Kohn, G., New cyclotetrasiloxanes bearing sila-alkyl substituted side chains and their applications as templates for gold nanowires. Dalton Transactions 2013, 42, (21), 7768-7774.

44. Zabetakis, K.; Ghann, W. E.; Kumar, S.; Daniel, M.-C., Effect of high gold salt concentrations on the size and polydispersity of gold nanoparticles prepared by an extended Turkevich-Frens method. Gold Bulletin 2012, 45, (4), 203-211.

45. Kumar, S.; Gandhi, K.; Kumar, R., Modeling of formation of gold nanoparticles by citrate method. Ind Eng Chem Res 2007, 46, 3128.

46. Grand, J.; Adam, P.-M.; Grimault, A.-S.; Vial, A.; Lamy de la Chapelle, M.; Bijeon, J.-L.; Kostcheev, S.; Royer, P., Optical Extinction Spectroscopy of Oblate, Prolate and Ellipsoid Shaped Gold Nanoparticles: Experiments and Theory. Plasmonics 2006, 1, (2), 135-140.

47. Halas, N. J.; Lal, S.; Chang, W.-S.; Link, S.; Nordlander, P., Plasmons in Strongly Coupled Metallic Nanostructures. Chemical Reviews 2011, 111, (6), 3913-3961.

48. Nordlander, P.; Oubre, C.; Prodan, E.; Li, K.; Stockman, M. I., Plasmon Hybridization in Nanoparticle Dimers. Nano Letters 2004, 4, (5), 899-903.

49. Garcia de Abajo, F. J., Nonlocal Effects in the Plasmons of Strongly Interacting Nanoparticles, Dimers, and Waveguides. The Journal of Physical Chemistry C 2008, 112, (46), 17983-17987. 
50. Romero, I.; Aizpurua, J.; Bryant, G. W.; Abajo, F. J. G. a. d., Plasmons in nearly touching metallic nanoparticles: singular response in the limit of touching dimers. Optics Express 2006, 14, (21), 9988-9999.

51. Lassiter, J. B.; Aizpurua, J.; Hernandez, L. I.; Brandl, D. W.; Romero, I.; Lal, S.; Hafner, J. H.; Nordlander, P.; Halas, N. J., Close Encounters between Two Nanoshells. Nano Letters 2008, 8, (4), $1212-1218$. 